\title{
CIRURGIAS REALIZADAS EM IDOSOS EM UM HOSPITAL PÚBLICO DO INTERIOR DE SÃO PAULO
}

\author{
Surgeries performed on elderly patients in a public hospital in the state of São Paulo
}

Cirugías realizadas en idosos en un hospital público del interior de São Paulo

\author{
Patrícia Santos Moreira' ๑, Léia Regina de Souza Alcântara ${ }^{2 *} \odot$, Jamille Duran Matilde $^{3} \oplus$, \\ Letícia Costa Rinaldi ${ }^{4} \odot$, Marla Andréia Garcia de Avila ${ }^{5}$ (อ)
}

RESUMO: Objetivo: Identificar a prevalência dos procedimentos cirúrgicos realizados em idosos em um centro cirúrgico de um hospital público do interior do estado de São Paulo e caracterizar tais procedimentos. Método: Estudo transversal, retrospectivo, quantitativo. A amostra constou de 7.483 procedimentos cirúrgicos em idosos, realizados entre 2013 e 2015. Os dados foram coletados a partir do sistema de cirurgia da instituição sede do estudo. Resultados: A faixa etária na qual prevaleceram os procedimentos foi entre 60 e 70 anos de idade; a maior média de tempo para recuperação da anestesia foi entre 71 e 80 anos. As especialidades que mais realizaram procedimentos foram: ortopedia, urologia e oftalmologia. As anestesias mais empregadas foram: geral inalatória, endovenosa, local com sedação e raquideana; 37,3\% usaram o serviço de anestesia, porém não estava descrito em prontuário qual foi o tipo de anestesia realizada. Os óbitos decorrentes dos procedimentos ou complicações das cirurgias foram 1.140; três deles ocorreram no centro cirúrgico e os demais, em enfermarias ou unidades de cuidados intensivos. Conclusão: Os dados aqui apresentados reforçam a necessidade de novos modelos de assistência, com melhorias da assistência multidisciplinar geriátrica, no atendimento perioperatório aos pacientes idosos.

Palavras-chave: Cirurgia. População idosa. Dinâmica populacional.

ABSTRACT: Objective: To identify the prevalence of surgical procedures performed in the elderly in a surgical center of a public hospital in the state of São Paulo and to characterize such procedures. Method: Cross-sectional, retrospective, quantitative study. The sample consisted of 7,483 surgical procedures performed in the elderly, between 2013 and 2015. Data were collected from the surgical information system of the institution under study. Results: The age range was between 60 and 70 years of age; the highest average anesthesia recovery time was between 71 and 80 years. The specialties that performed the most procedures were: orthopedics, urology and ophthalmology. The most commonly used anesthesias were: general inhalation, intravenous, local with sedation and spinal; $37,3 \%$ used anesthesia, however the type was not described in the patient medical records. There were 1,140 deaths resulting from the procedures or complications; three of them occurred in the surgical center, while the others occured in the intensive care unit or ward. Conclusion: The data presented here reinforce the need for new models of care, with improvements in geriatric multidisciplinary care, in perioperative care for the elderly. Keywords: Surgery. Elderly population. Population dynamics.

RESUMEN: Objetivo: Identificar la prevalencia de los procedimientos quirúrgicos realizados en ancianos en un centro quirúrgico de un hospital público del interior del estado de São Paulo y caracterizar tales procedimientos. Método: Estudio transversal, retrospectivo, cuantitativo. La muestra constató de 7.483 procedimientos quirúrgicos en ancianos, realizados entre 2013 y 2015. Los datos fueron recolectados a partir del sistema de cirugía de la institución sede del estudio. Resultados: El grupo de edad en el que prevalecieron los procedimientos fue entre 60 y 70 años de edad; la mayor media de tiempo para la recuperación de la anestesia fue entre 71 y 80 años. Las especialidades que más realizaron procedimientos fueron: ortopedia, urología y oftalmología. Las anestesias más empleadas fueron: general inhalatoria, endovenosa, local con sedación y raquídea; $37,3 \%$ usaron el servicio de anestesia, pero no estaba descrito en prontuario cuál fue el tipo de anestesia realizada. Las muertes derivadas de los procedimientos o complicaciones de las cirugías fueron 1.140; Y tres de ellos ocurrieron en el centro quirúrgico y los demás, en enfermerías o unidades de cuidados intensivos. Conclusión: Los datos aquí presentados refuerzan la necesidad de nuevos modelos de asistencia, con mejoras de la asistencia multidisciplinaria geriátrica, en la atención perioperatoria a los pacientes ancianos. Palabras clave: Cirugía. Poplación anciana. Dinámica poblacional.

'Enfermeira. Residente em Saúde do Adulto e do Idoso pela Universidade Estadual Paulista “Júlio de Mesquita Filho” (Unesp) - Botucatu (SP), Brasil. Enfermeira, especialista em Enfermagem em Centro Cirúrgico, Recuperação Pós-Anestésica e Central de Material e Esterilização; mestre em Ciências da Saúde. Docente da disciplina Enfermagem Perioperatória do curso de Enfermagem da Universidade Estadual do Norte do Paraná (UENP) - Bandeirantes (PR), Brasil.

${ }^{3}$ Enfermeira. Residente do Programa de Residência Multiprofissional em Saúde do Adulto e do Idoso no Hospital das Clínicas da Faculdade de Medicina de Botucatu, do Departamento de Enfermagem da UNESP - Botucatu (SP), Brasil.

${ }^{4}$ Graduanda em Enfermagem pela Unesp - Botucatu (SP), Brasil.

${ }^{5}$ Enfermeira; mestre em Biotecnologia Médica; doutora em Saúde Coletiva. Orientadora do Programa de Pós-Graduação em Enfermagem da UNESP - Botucatu (SP), Brasil.

*Autor correspondente: alcantara@uenp.edu.br

Recebido: 29/07/2018 - Aprovado: 01/05/2019

DOl: 10.5327/Z1414-4425201900020004 


\section{INTRODUÇÃO}

A transição demográfica é um fenômeno de ocorrência mundial, no qual, progressivamente, o número de jovens decresce e há aumento considerável da população com 60 anos de idade ou mais. Igualmente, o Brasil tem vivenciado o aumento da população dessa faixa etária. Segundo o Instituto Brasileiro de Geografia e Estatística (IBGE), o índice de envelhecimento elevou-se de $31,7 \%$ em 2001 para $51,8 \%$ em 2011. Isso demonstra que há, em média, uma pessoa de 60 anos ou mais para cada duas pessoas com menos de 15 anos $^{1}$. Estima-se que em 2050 existirão 2 milhões de idosos no mundo, e que no Brasil serão cerca de 28 milhões de idosos ${ }^{2}$.

$\mathrm{O}$ aumento da esperança de vida se deve à redução da mortalidade infantil, que progressivamente atingiu todas as idades, incluindo as taxas de mortalidade da população idosa, que tem experimentado grande queda. Deve-se, também, aos avanços das tecnologias na área da saúde e ao desenvolvimento de políticas públicas voltadas aos idosos ${ }^{3}$.

O envelhecimento é um processo biológico irreversível, natural e individual. O termo envelhecimento é marcado por mudanças de forma e função ao longo da vida, que ocorrem nos organismos após a maturação sexual e que, progressivamente, comprometem a funcionalidade, a mobilidade e a independência, influenciando a capacidade de resposta dos idosos ao estresse ambiental e à manutenção da homeostasia, bem como pela alta prevalência de doenças crônico-degenerativas, que levarão essa população a necessitar de assistência qualificada dos serviços de saúde em todos os níveis de atenção ${ }^{4}$.

Em consequência de uma maior expectativa de vida, tratamentos e cirurgias tendem a ser mais rotineiros. A Organização Mundial de Saúde (OMS) prevê que mais de 60 milhões de pessoas passarão por cirurgias por motivos traumáticos e mais de 30 milhões para tratar doenças malignas a cada ano ${ }^{5}$. Complicações cirúrgicas são mais comuns na população idosa por causa do organismo lentificado, da mobilidade física prejudicada, da maior probabilidade de infecções e das dificuldades advindas das próprias doenças de base, tornando necessário o preparo dos profissionais para cuidar de pacientes geriátricos ${ }^{5}$.

A literatura traz poucos estudos considerando o perfil das cirurgias realizadas em idosos, bem como as características dessa população. Em geral, os estudos que avaliam os procedimentos cirúrgicos em idosos mostram cirurgias realizadas em segmentos únicos ${ }^{6-8}$. A gestão hospitalar aparece focando os custos das cirurgias, especificamente os das cirurgias oncológicas, analisando o levantamento de ações para diminuir os custos e os repasses dos convênios?.

Considerando o aumento da esperança de vida na população, da escassez de trabalhos relacionados ao tema e da importância da assistência à saúde dos idosos em todos os segmentos, propomos a realização deste estudo, visando trazer subsídios para melhoria na assistência aos idosos no centro cirúrgico (CC) e a gestão da unidade de CC.

\section{OBJETIVO}

Identificar os procedimentos cirúrgicos realizados em idosos em um CC de um hospital público do interior do estado de São Paulo e caracterizar tais procedimentos.

\section{MÉTODOS}

Trata-se de um estudo transversal, retrospectivo, com abordagem quantitativa, não probabilística.

O presente estudo foi conduzido no Hospital das Clínicas (HC) da cidade de Botucatu, interior do estado de São Paulo. Estima-se que a abrangência populacional de atendimento hospitalar seja de 1,5 milhão de usuários, provenientes de 68 municípios do Departamento Regional de Saúde de Bauru (DRS VI). O HC conta com 385 leitos, sendo 52 de terapia intensiva (30 adultos, 15 neonatais e 7 pediátricos), 198 consultórios médicos e 31 salas especializadas. Anualmente, a instituição realiza, em média, 2 milhões de exames, 650 mil consultas e 25 mil internações.

É considerada a maior unidade pública de saúde da região de Botucatu, vinculada ao Sistema Único de Saúde (SUS). O Hospital das Clínicas da Faculdade de Medicina de Botucatu (HCFMB) tem em seu quadro 170 enfermeiros e mais de 800 técnicos de enfermagem.

O CC da instituição é constituído de 13 salas operatórias, que atendem a cirurgias de porte I, II, III e IV, de diversas especialidades, de acordo com o horário semanal estabelecido para cada equipe. Atuam no CC aproximadamente 13 equipes cirúrgicas, anestesiologistas e equipe de enfermagem, entre outros profissionais. No ano de 2014 o serviço realizou 8.967 cirurgias.

Os dados foram coletados a partir do sistema de cirurgia do serviço. Foram incluídos os procedimentos que aconteceram no CC do HCFMB, nos anos de 2013 a 2015, em usuários com 60 anos ou mais. Foram excluídos os procedimentos cujos 
dados estavam incompletos no prontuário. As variáveis analisadas foram: sexo (masculino e feminino), idade (em anos), cirurgia realizada (classificada de acordo com a nomenclatura do Sistema de Gerenciamento de Tabelas de Procedimentos, Medicamentos e Órteses e Próteses e Materiais Especiais do SUS - SIGTAP), anestesia realizada, tempo de internação (em dias), especialidade médica, tempo cirúrgico (em minutos) e ocorrência de óbito durante a cirurgia.

Os resultados foram apresentados por estatística descritiva, com frequências absolutas e percentuais para as variáveis categorizadas, média e mediana. Os dados foram inseridos e tabulados em planilha Microsoft Excel e estão apresentados na forma de tabelas.

O projeto foi submetido para apreciação do Comitê de Ética em Pesquisa (CEP) da Faculdade de Medicina de Botucatu, sob o Parecer $\mathrm{n}^{\circ} 1.526 .015$, de 3 de maio de 2016. Foi solicitada e autorizada a dispensa do Termo de Consentimento Livre e Esclarecido, por se tratar da utilização de dados secundários.

\section{RESULTADOS}

A amostra do estudo foi composta dos dados de 7.483 procedimentos cirúrgicos em idosos, acima de 60 anos, entre os anos de 2013 e 2015 , e $51 \%$ foram realizados em pessoas do sexo masculino. A faixa etária na qual mais ocorreram procedimentos foi entre 60 e 70 anos de idade, em ambos os sexos. O tempo médio cirúrgico foi maior nessa faixa etária, regredindo nas demais faixas.

A recuperação da anestesia teve sua maior média de tempo na faixa etária dos 71 aos 80 anos de idade, seguida pela faixa etária dos 60 aos 70 anos. A média de internação prevaleceu na faixa etária dos 60 aos 70 anos (Tabela 1).

Ao analisar a variável especialidades cirúrgicas, verificou-se que os procedimentos ortopédicos foram os mais prevalentes, seguidos pela urologia, oftalmologia, cirurgia vascular, gastrocirurgia, neurocirurgia, cirurgia cardíaca, otorrinolaringologia, cirurgia geral e cirurgia torácica. A Tabela 2 mostra os procedimentos divididos por faixa etária.

A Tabela 3 aponta os principais procedimentos cirúrgicos realizados em pacientes, na faixa etária estudada.

A Tabela 4 mostra a prevalência das anestesias realizadas nos procedimentos cirúrgicos. Pode-se verificar que a anestesia geral inalatória e a anestesia endovenosa foram as mais empregadas, seguidas pela local com sedação e raquianestesia. Destaca-se que 37\% dos cirurgiões utilizaram o serviço de anestesia, porém não o descreveram no prontuário do paciente.

A análise dos óbitos decorrentes dos procedimentos ou de complicações das cirurgias mostrou um total de 1.140 mortes de pacientes idosos, e três delas ocorreram no CC e as demais, em enfermarias ou unidades de tratamento intensivo. A Tabela 5 nos mostra as características dos pacientes que foram a óbito.

Tabela 1. Distribuição das cirurgias segundo sexo, tempo de cirurgia, tempo de recuperação da anestesia e dias de internação.

\begin{tabular}{|c|c|c|c|c|c|}
\hline Variáveis & $\begin{array}{c}60 \text { a } 70 \text { anos } \\
n(\%)\end{array}$ & $\begin{array}{c}71 \text { a } 80 \text { anos } \\
n(\%)\end{array}$ & $\begin{array}{c}81 \text { a } 90 \text { anos } \\
n(\%)\end{array}$ & $\begin{array}{c}91 \text { anos ou mais } \\
n(\%)\end{array}$ & Total \\
\hline \multicolumn{6}{|l|}{ Sexo } \\
\hline Feminino & $1.930(47)$ & $1.081(49)$ & $575(56)$ & $94(60)$ & 3.680 \\
\hline Masculino & $2.180(53)$ & $1.117(51)$ & $444(44)$ & $62(40)$ & 3.803 \\
\hline Total & $4.110(100)$ & $2.198(100)$ & $1.019(100)$ & $156(100)$ & 7.483 \\
\hline \multicolumn{6}{|c|}{ Indicadores cirúrgicos } \\
\hline \multicolumn{6}{|c|}{ Tempo cirúrgico (minutos) } \\
\hline Média & 128 & 114 & 101 & 83 & 106,5 \\
\hline Mediana & 107 & 99,5 & 97 & 50 & 98,2 \\
\hline \multicolumn{6}{|c|}{ Tempo de recuperação da anestesia (minutos) } \\
\hline Média & 80,5 & 88,5 & 84 & 88,5 & 85,3 \\
\hline Mediana & 94,5 & 284 & 61 & 66 & 172,5 \\
\hline \multicolumn{6}{|c|}{ Tempo de internação (dias) } \\
\hline Média & 12,5 & 12 & 12 & 9 & 11,3 \\
\hline Mediana & 16 & 14 & 13,5 & 9 & 13,7 \\
\hline
\end{tabular}


Tabela 2. Distribuição das cirurgias segundo a especialidade e faixa etária.

\begin{tabular}{|c|c|c|c|c|c|}
\hline $\begin{array}{l}\text { Variáveis } \\
\text { Especialidades }\end{array}$ & $\begin{array}{c}60 \text { a } 70 \text { anos } \\
n(\%)\end{array}$ & $\begin{array}{c}71 \text { a } 80 \text { anos } \\
n(\%)\end{array}$ & $\begin{array}{c}81 \text { a } 90 \text { anos } \\
n(\%)\end{array}$ & $\begin{array}{c}91 \text { anos ou mais } \\
n(\%)\end{array}$ & $\begin{array}{l}\text { Total } \\
\text { n (\%) }\end{array}$ \\
\hline Ortopedia & $674(16)$ & 370 (17) & $278(27)$ & $63(40)$ & $1.385(18,5)$ \\
\hline Urologia & $578(14)$ & $227(10)$ & $54(5)$ & $10(6)$ & $869(11,6)$ \\
\hline Oftalmologia & $382(9)$ & $332(15)$ & $142(14)$ & $8(5)$ & $864(11,5)$ \\
\hline Vascular & $405(10)$ & $302(14)$ & $121(12)$ & $22(14)$ & $850(11,4)$ \\
\hline Gastrocirurgia & $458(11)$ & $234(11)$ & $107(10)$ & $10(6)$ & $809(10,8)$ \\
\hline Neurocirurgia & $368(9)$ & $152(7)$ & $57(6)$ & $9(8)$ & $586(7,8)$ \\
\hline Cirurgia cardíaca & $221(5)$ & $140(6)$ & $80(8)$ & $12(8)$ & $453(6,2)$ \\
\hline Otorrinolaringologia & $215(5)$ & $104(5)$ & $41(4)$ & $4(3)$ & $364(4,9)$ \\
\hline Cirurgia geral & $171(4)$ & $75(3)$ & $39(4)$ & $11(7)$ & $286(3,8)$ \\
\hline Cirurgia torácica & $126(3)$ & $52(2)$ & $12(1)$ & $1(0,64)$ & $191(2,6)$ \\
\hline Outras especialidades & - & - & - & - & $816(10,9)$ \\
\hline
\end{tabular}

Tabela 3. Distribuição dos principais procedimentos cirúrgicos realizados em idosos.

\begin{tabular}{|l|c|c|}
\hline Procedimentos cirúrgicos & $\mathbf{n}$ & $\mathbf{( \% )}$ \\
\hline Tratamento cirúrgico de fratura proximal de fêmur & 270 & 17,6 \\
\hline Laparotomia exploradora & 259 & 16,8 \\
\hline Traqueostomia & 182 & 11,8 \\
\hline Facectomia com implante de lente intraocular & 157 & 10,2 \\
\hline Amputação/desarticulação de dedo & 128 & 8,3 \\
\hline Debridamento de úlcera/tecido desvitalizado & 125 & 8,1 \\
\hline Ressecção endoscópica de próstata & 119 & 7,7 \\
\hline Embolectomia arterial & 114 & 7,4 \\
\hline Colecistectomia & 97 & 6,3 \\
\hline Histerectomia vaginal & 87 & 5,7 \\
\hline
\end{tabular}

Tabela 4. Distribuição das cirurgias segundo anestesias realizadas nos pacientes idosos.

\begin{tabular}{|l|c|c|}
\hline Tipos de anestesias & $\mathbf{n}$ & $\mathbf{( \% )}$ \\
\hline Geral inalatória e endovenosa & 2.012 & 26,9 \\
\hline Local/sedação & 1.221 & 16,3 \\
\hline Raquianestesia & 1.172 & 15,7 \\
\hline Bloqueios & 237 & 3,2 \\
\hline Peridural & 51 & 0,7 \\
\hline Não informado & 2.790 & 37,3 \\
\hline Total & 7.483 & 100,0 \\
\hline
\end{tabular}

Tabela 5. Características dos óbitos dos pacientes idosos.

\begin{tabular}{|l|c|c|c|}
\hline Óbito & $\begin{array}{c}\text { Intraoperatório } \\
\mathbf{n}\end{array}$ & $\begin{array}{c}\text { Pós-operatório } \\
\mathbf{n}\end{array}$ & Total \\
\hline Período & 3 & 1.137 & 1.140 \\
\hline Sexo & & & \\
\hline Feminino & 0 & 472 & 472 \\
\hline Masculino & 3 & 665 & 668 \\
\hline Média de internação (dias) & 10 & 13 & - \\
\hline Tempo de recuperação da anestesia (minutos) & - & 92 & - \\
\hline Anestesia prevalente & Geral & Geral & - \\
\hline Especialidade prevalente & Cardíaca/vascular & Vascular/gastrocirurgia/ \\
\hline Tempo de cirurgia (minutos) & 376 & neurocirurgia \\
\hline Idade (anos) & 79 & 135 \\
\hline
\end{tabular}




\section{DISCUSSÃO}

A amostra deste estudo foi composta dos dados de 7.843 pacientes idosos, submetidos a procedimentos cirúrgicos entre os anos 2013 e 2015.

O aumento da população idosa no Brasil e no mundo evidencia maior procura dos hospitais e, consequentemente, maior entrada nos $\mathrm{CC}^{10}$. Quando se analisa o total de procedimentos cirúrgicos, não houve prevalência significativa do sexo masculino ou feminino, porém, quando se faz a análise das faixas etárias entre 60 e 80 anos, verifica-se prevalência do sexo masculino. Tal ocorrência nos leva a crer que o autocuidado pode ser mais deficiente entre os homens, levando a maiores taxas de internações e cirurgias.

O tempo médio e mediano dos procedimentos cirúrgicos não apresentou grandes variações entre as faixas etárias, porém convém ressaltar que o tempo cirúrgico está diretamente relacionado a diversas complicações, tais como infecção de sítio cirúrgico, que tem sua probabilidade aumentada em 13,17 e $37 \%$ para cada 15,30 e 60 minutos de cirurgia, respectivamente ${ }^{11}$.

O tempo de internação também não apresentou variações significativas entre as faixas etárias. As comorbidades comuns nas faixas etárias estudadas podem prolongar o tempo de hospitalização, tornando o indivíduo vulnerável a eventos adversos que pioram o prognóstico e oneram os hospitais ${ }^{12}$.

O envelhecimento como processo biológico traz o declínio das funções orgânicas e a ocorrência de doenças crônico-degenerativas, como consequência das alterações morfológicas, bioquímicas e funcionais. Estas poderão levar o indivíduo, em algum momento de senilidade, a vivenciar uma situação cirúrgica. Assim, pode-se verificar (Tabelas 2 e 3) que as especialidades cirúrgicas, bem como os procedimentos realizados, envolvem todos os sistemas orgânicos. Todavia, o estilo de vida e os hábitos podem ditar a qualidade do processo de envelhecimento; portanto, é de grande importância que a senilidade seja pensada e preparada, desde as mais tenras fases da vida ${ }^{13}$.

Destaque pode ser dado à alta prevalência de procedimentos cirúrgicos ortopédicos (Tabela 2), e a maioria foi por fratura de fêmur (Tabela 3). Estudo conduzido nos países da União Europeia mostrou que em 2010 ocorreram 610 mil fraturas de quadril/fêmur em idosos com mais de $70 \operatorname{anos}^{14} \mathrm{e}$, no Brasil, entre 2008 e 2012, foram 38.755 fraturas de fêmur, de modo que essa ocorrência vem aumentando nas últimas décadas ${ }^{2}$. As fraturas são resultantes de quedas e fragilidades ósseas e têm como consequência a perda da independência e os altos índices de morbidade e mortalidade, elevando os custos para o sistema de saúde ${ }^{15}$. Diante dessa realidade, ressaltamos a importância da implementação das medidas preventivas recomendadas pela $\mathrm{OMS}^{16}$.

A anestesia geral inalatória e a anestesia endovenosa foram as mais aplicadas nos pacientes idosos. Estudo questiona se a anestesia geral pode ser prejudicial ao cérebro do idoso, visto que, não raramente, pacientes idosos apresentam deterioração da função cognitiva pós-operatória, podendo levar ao aumento da morbidade e da mortalidade ${ }^{17}$. A raquianestesia, assim como as demais modalidades anestésicas, apresenta vantagens sobre a anestesia geral, tais como: variáveis hemodinâmicas estáveis, menor perda de sangue, menos dor pós-operatória, tempo de recuperação mais rápido e menos confusão pós-operatória; porém o bloqueio simpático produzido pode resultar em hipotensão, bradicardia e paradas cardíacas ${ }^{18}$.

Chamou-nos a atenção o alto índice de equipes cirúrgicas que utilizaram o serviço de anestesia para os pacientes idosos, porém não tiveram os dados devidamente preenchidos no Prontuário Eletrônico do Paciente (PEP). Embora a qualidade do preenchimento não tenha sido objetivo deste estudo, convém ressaltar a importância desse achado, uma vez que o PEP visa contribuir para a eficiência e a qualidade da atenção, integrar organizações de saúde e facilitar a sua gestão e pesquisa ${ }^{19}$. O fato de não haver registro em prontuário do tipo de anestesia empregado em determinados procedimentos cirúrgicos é um dado significativo, por conta da importância dessa informação para o planejamento e a implemantação dos cuidados perioperatórios.

Verificou-se uma frequência de óbitos de $14,53 \%$. Estudo que avaliou a mortalidade em pacientes idosos com fratura de quadril revelou uma taxa de $11,9 \%$ de óbitos durante a internação, relacionados a comorbidades, infecções no período de internação e tempo entre internação e cirurgia superior a 7 dias $^{20}$. O tempo de cirurgia e a idade também foram maiores nos óbitos que ocorreram no período intraoperatório, ou seja, dentro do CC, reafirmando o risco de cirurgias longas, principalmente em pacientes com idades mais avançadas. Procedimentos de longa duração podem servir de marcadores de complexidade dos casos.

Considerando-se os óbitos pós-cirúrgicos, seja nas unidades de internação, seja nas unidades de tratamento intensivo, as cirurgias vasculares continuaram em primeiro lugar, seguidas da gastrocirurgia e da neurocirurgia, especialidades de grande complexidade em nível cirúrgico. 
Em função do aumento de doenças cardiovasculares na população em estudo, por toda carga genética e hábitos de vida incompatíveis com suas doenças de base, as complicações intraoperatórias e pós-operatórias aumentam e, consequentemente, a mortalidade também tem seus índices elevados ${ }^{10}$.

\section{CONCLUSÃO}

O presente estudo identificou as principais causas que levam pacientes idosos a necessitar de procedimentos cirúrgicos e que, para aperfeiçoar o desenvolvimento do serviço, é importante entender o padrão de mudança de cuidados cirúrgicos para esse grupo de pacientes. Os dados aqui apresentados reforçam a necessidade de novos modelos de atendimento, com vistas à melhoria da assistência multidisciplinar geriátrica no atendimento pré-operatório prestado aos idosos. A melhoria da qualidade de preenchimento do prontuário eletrônico também é fundamental para novas pesquisas. Documentos eletrônicos específicos para a anestesia e para a recuperação imediata podem proporcionar maior segurança para o profissional e para o paciente, além de contribuir para atividades acadêmicas e de pesquisa.

Este estudo tem como limitações a coleta de dados retrospectivos, uma amostra não probabilística e realizada em um único contexto. Ademais, os resultados aqui encontrados não podem ser generalizados, devendo ser realizados estudos na temática com outros desenhos metodológicos e outras amostras de pacientes idosos.

\section{REFERÊNCIAS}

1. Brasil. Ministério do Planejamento Orçamento e Gestão. Instituto Brasileiro de Geografia e Estatística. Pesquisa Nacional por Amostra de Domicílios. Rio de Janeiro: Ministério do Planejamento Orçamento e Gestão / Instituto Brasileiro de Geografia e Estatística; 2012.

2. Soares DS, de Mello LM, da Silva AS, Martinez EZ, Nunes AA. Fraturas de fêmur em idosos no Brasil: análise espaço-temporal de 2008 a 2012. Cad Saúde Pública. 2014;30(12):2669-78. http://dx.doi. org/10.1590/0102-311x00218113

3. Rohde G, Haugeberg G, Mengshoel AM, Moum T, Wahl AK. Two-year changes in quality of life in elderly patients with low-energy hip fractures: a case-control study. BMC Musculoskelet Disord [Internet]. 2010 [acessado em 26 mar. 2018];11:226. Disponível em: http://bmcmusculoskeletdisord. biomedcentral.com/articles/10.1186/1471-2474-11-226. https://doi. org/10.1186/1471-2474-11-226

4. Strobl R, Müller M, Emeny R, Peters A, Grill E. Distribution and determinants of functioning and disability in aged adults: results from the German KORA-Age study. BMC Public Health [Internet]. 2013 [acessado em 26 mar. 2018];13:137. Disponivel em: http://bmcpublichealth. biomedcentral.com/articles/10.1186/1471-2458-13-137. https:// doi.org/10.1186/1471-2458-13-137

5. Santana RF, do Amaral DM, Pereira SK, Delphino TM, Cassiano KM. The occurrence of the delayed surgical recovery nursing diagnosis among adults and the elderly. Acta Paul Enferm. 2014;27(1):35-9. http://dx.doi.org/10.1590/1982-0194201400008

6. Sierink JC, de Castro SMM, Russell NS, Geenen MM, Steller EP, Vrouenraets BC. Treatment strategies in elderly breast cancer patients: Is there a need for surgery? Breast [Internet]. 2014 [acessado em 26 mar. 2018];23(6):793-8. Disponível em: http://www.ncbi.nlm.nih.gov/ pubmed/25212636. https://doi.org/10.1016/j.breast.2014.08.006

7. Moug SJ, McCarthy K, Coode-Bate J, Stechman MJ, Hewitt J. Laparoscopic versus open surgery for colorectal cancer in the older person: a systematic review. Ann Med Surg [lnternet]. 2015 [acessado em 26 mar. 2018];4(3):311-8. Disponivel em: http://linkinghub.elsevier. com/retrieve/pii/S2049080115000795. https://doi.org/10.1016/j. amsu.2015.08.002

8. O'Lynnger TM, Zuckerman SL, Morone PJ, Dewan MC, VasquezCastellanos RA, Cheng JS. Trends for spine surgery for the elderly: implications for access to healthcare in North America. Neurosurgery [Internet]. 2015 [acessado em 26 mar. 2018];77(Supl. 1):S136-41. Disponível em: https://academic.oup.com/neurosurgery/articlelookup/doi/10.1227/NEU.0000000000000945. https://doi.org/10.1227/ NEU.0000000000000945

9. Nathan $\mathrm{H}, \mathrm{Atoria} \mathrm{CL}, \mathrm{Bach} \mathrm{PB}$, Elkin EB. Hospital volume, complications, and cost of cancer surgery in the elderly. J Clin Oncol [Internet]. 2015 [acessado em 26 mar. 2018];33(1):107-14. Disponível em: http:// ascopubs.org/doi/10.1200/JC0.2014.57.7155. https://doi.org/10.1200/ JCO.2014.57.7155

10. Mendoza IYQ, Grazziano ES. Promoção de práticas seguras no cuidado perioperatório do idoso. In: Grazziano SDE, Viana LD, Harada SCJDM, Pedreira GLDM, editores. Enfermagem perioperatória e cirurgia segura. São Caetano do Sul: Yendis; 2015. p. 219-235.

11. Cheng H, Chen BP-H, Soleas IM, Ferko NC, Cameron CG, Hinoul P. Prolonged operative duration increases risk of surgical site infections: a systematic review. Surg Infect (Larchmt) [Internet]. 2017 [acessado em 26 mar. 2018];18(6):722-35. Disponível em: http://www.ncbi.nlm.nih.gov/pubmed/28832271. https://doi. org/10.1089/sur.2017.089

12. de Freitas FAZ, Santos ES de S, Pereira LSM, Lustosa LP. Vulnerabilidade física de idosos na alta hospitalar. Fisioter e Pesqui [Internet]. 2017 [acessado em 28 mar. 2018];24(3):2538. Disponível em: http://www.scielo.br/scielo.php?script=sci_ arttext\&pid=S1809-29502017000300253\&lng=pt\&tlng=pt. https:// doi.org/10.1590/1809-2950/16205224032017 
13. Barbon FJ, Wiethölter P, Flores RA. Alterações celulares no envelhecimento humano. J Oral Investig [Internet]. 2016 [acessado em 28 mar. 2018];5(1):61-5. Disponível em: http://www.bibliotekevirtual. org/index.php/2013-02-07-03-02-35/2013-02-07-03-03-11/2010-joi/ v05n01/20634-alteracoes-celulares-no-envelhecimento-humano.html. http://dx.doi.org/10.18256/2238-510X/j.oralinvestigations.v5n1 p61-65

14. Hernlund E, Svedbom A, Ivergård M, Compston J, Cooper C, Stenmark $\mathrm{J}$, et al. Osteoporosis in the European Union: medical management, epidemiology and economic burden: a report prepared in collaboration with the International Osteoporosis Foundation (IOF) and the European Federation of Pharmaceutical Industry Associations (EFPIA). Arch Osteoporos. 2013;8:136. https://doi.org/10.1007/s11657-013-0136-1

15. Loures FB, Chaoubah A, de Oliveira VM, Almeida AM, Campos EM de $\mathrm{S}$, de Paiva EP. Economic analysis of surgical treatment of hip fracture in older adults. Rev Saúde Pública. 2015;49:12. https://dx.doi.org/1 0.1590\%2FS0034-8910.2015049005172

16. de Avila MAG, Pereira GJC, Bocchi SCM. Cuidadores informais de idosos em pós-operatório de cirurgia de fêmur proximal: prevenção de novas quedas. Ciên Saúde Colet [Internet]. 2015 [acessado em 30 mar. 2018];20(6):1901-7. Disponível em: http://www.scielo.br/scielo. php?script=sci_arttext\&pid=S1413-81232015000601901\&lng=pt\&t lng=pt. http://dx.doi.org/10.1590/1413-81232015206.17202014
17. Purdon PL, Pavone KJ, Akeju O, Smith AC, Sampson AL, Lee J, et al. The ageing brain: age-dependent changes in the electroencephalogram during propofol and sevoflurane general anaesthesia. $\mathrm{Br} J$ Anaesth [Internet]. 2015 [acessado em 31 mar. 2018];115(Supl. 1):i46-57. Disponível em: http://www.ncbi.nlm.nih.gov/pubmed/26174300. https://doi.org/10.1093/bja/aev213

18. Mung'ayi V, Mbaya K, Sharif T, Kamya D. A randomized controlled trial comparing haemodynamic stability in elderly patients undergoing spinal anaesthesia at $L 5, S 1$ versus spinal anaesthesia at $L 3,4$ at a tertiary African hospital. Afr Health Sci [Internet]. 2015 [acessado em 31 mar. 2018];15(2):466-79. Disponivel em: http://www.ncbi.nlm. nih.gov/pubmed/26124793. https://doi.org/10.4314/ahs.v15i2.21

19. Pinto MLR, Jorge MJ. A qualidade da informação do prontuário eletrônico do paciente: um estudo de caso sobre avaliação da inovação. Vita et Sanitas. 2014;(8):58-80.

20. Edelmuth SVCL, Sorio GN, Sprovieri FAA, Gali JC, Peron SF. Comorbidades, intercorrências clínicas e fatores associados à mortalidade em pacientes idosos internados por fratura de quadril. Rev Bras Ortop [Internet]. 2018 [acessado em 31 mar. 2018];53(5):543-51. Disponível em: https://www.sciencedirect.com/ science/article/pii/S0102361617303958. https://doi.org/10.1016/j. rbo.2017.07.009 